\title{
Innovación tecnocientífica para el fortalecimiento del E-government una apreciación desde el enfoque de los estudios de la ciencia - tecnología - sociedad
}

DOI: https://doi.org/10.33262/ap.v3i3.2.105

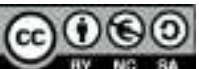

Techno-scientific innovation for the strengthening of E-government an appreciation from the approach of science - technology - society studies

\author{
José Fabián Fonseca Vasconez. ${ }^{1}$, Carmita Efigenia Andrade Álvarez. ${ }^{2}$ \& \\ Jimena Catalina Viteri Ojeda. ${ }^{3}$
}

\begin{abstract}
.
Introduction The COVID 19 pandemic forced digitalization and techno-scientific innovation in order to generate development in the face of the slowdown in human mobility, in this area the present research is based on the existence of dissatisfaction with the services on the part of the clients of the decentralized autonomous governments (GADs) of the province of Chimborazo. The objective of this research is to design an action plan based on science - technology - society studies to increase the satisfaction of E-government clients, for which a review of the literature on projects, local development, and techno-scientific innovation, which allowed the authors a better understanding of the subject. Methodology, a document review guide, an observation guide and a satisfaction survey were applied to the clients of the project that provided valuable information for the diagnosis of the current situation of the main causes and difficulties. Results, all the information collected during the investigation allowed the design of a plan that is adjusted to the main needs to reverse the existing situation. Conclusions The systematization of the theoretical references and the analysis of customer satisfaction made it possible to determine that techno-scientific innovation is a transversal axis and not a pillar of

\footnotetext{
${ }^{1}$ Universidad Técnica de Babahoyo, Facultad Administración Finanzas e Informática, Quevedo, Ecuador, jfonsecav@ubq.edu.ec, https://orcid.org/0000-0003-3975-8751

${ }_{2}^{2}$ Escuela Superior Politécnica del Chimborazo, Facultad de Administración de Empresas. Chimborazo, Ecuador, candrade_a@espoch.edu.ec, https://orcid.org/0000-0003-4507-425X

3 Escuela Superior Politécnica del Chimborazo, Sede Orellana. Morona Santiago, Ecuador, jcviteri@espoch.edu.ec, https://orcid.org/0000-0001-7049-8981
} 
development, considering that technology is another productive factor.

Key words: Innovation, E-government science, technology, society

\section{Resumen}

Introducción la pandemia por la COVID 19 obligo a la digitalización e innovación tecno científica en post de generar desarrollo ante la desaceleración de movilidad humana, en este ámbito la presente investigación parte de la existencia de insatisfacciones con los servicios por parte de los clientes de los gobiernos autónomos descentralizados ( GADs )de la provincia de Chimborazo. El objetivo de esta investigación es diseñar un plan de acciones a partir de los estudios de la ciencia - tecnología - sociedad para elevar la satisfacción de los clientes del E-government para lo cual se hizo una revisión de la literatura sobre proyectos, desarrollo local, e innovación tecno científica, lo cual permitió a los autores una mejor comprensión alrededor del tema. Metodología, se aplicó una guía de revisión de documentos, una guía de observación y una encuesta de satisfacción a los clientes del proyecto que brindaron información valiosa para el diagnóstico de la situación actual de las principales causas y dificultades. Resultados, toda la información recopilada durante la investigación permitió el diseño de un plan que se ajusta a las principales necesidades para revertir la situación existente. Conclusiones la sistematización de los referentes teóricos y el análisis de la satisfacción de los clientes permitieron determinar que la innovación tecno científica es un eje transversal y no un pilar del desarrollo considerando que la tecnología es un factor productivo más.

Palabras clave: Innovación, E-government ciencia, tecnología, sociedad

\section{Introducción}

Cada vez, es más habitual escuchar hablar sobre la importancia que tiene para los GADs contar procesos tecnológicos que permitan agilitar los tramites y disminuir los tiempos en cada proceso promoviendo eficiencia y eficacia para el usuario. Con distinto nivel de resultados alcanzados, recursos, planificación y participación de la comunidad, etc. muchas localidades han estado implementando, en Ecuador, se crea en 2009 el Plan de Gobierno Electrónico (PGE) como un instrumento para la modernización del Estado. Este plan tiene como objetivo sumar esfuerzos institucionales para crear formas pioneras e innovadoras de interacción entre el Gobierno y la sociedad civil ecuatoriana. Los principales factores que impulsaron a los gobiernos locales a asumir un nuevo rol fueron el traspaso de algunas funciones del Estado Nacional hacia las provincias cantones y parroquias, el aumento del desempleo y de la pobreza y la emergencia de nuevas demandas de la sociedad civil hacia los gobiernos locales.

En este proceso se inscribe el desarrollo local a partir de la innovación tecno científica, constituyéndose en un planteamiento mundial y con énfasis en nuestro país sobre la necesidad de los procesos tecnológicos y para generar mayor dinámica social de los espacios territoriales para contribuir al desarrollo nacional. 
A nivel territorial se han materializados diversos proyectos informáticos de desarrollo local donde cada GADs cuenta proyectos de digitalización de servicios

Los GADs tiene como objeto general fomentar el desarrollo sostenible a través de la articulación social, económica ambiental e institucional; y dentro de sus objetivos específicos esta la articulación informática

Existen beneficios del E-government como dinamizador de Desarrollo Local pero aun así persisten insatisfacciones en los clientes en cuanto a: la calidad de los productos y servicios que oferta, por la poca aplicabilidad practica de los mismos por tanto no existe correspondencia entre la demanda del cliente y la capacidad ofrecida, es decir no se logra satisfacer las demandadas poblacionales,

Los autores de la investigación definen que la situación problemática de la misma radica en que existen insatisfacciones con los servicios digitales por parte de los clientes que utilizan la plataforma oficial de los GADs de la provincia de Chimborazo

De ahí que se manifieste el siguiente problema de investigación: ¿Cómo contribuir con la elevación de la satisfacción de los clientes que están haciendo uso de E-government?

Para dar solución a dicho problema se desarrolla la investigación que tiene como objetivo general: diseñar un plan de acciones a partir de los estudios de la ciencia - tecnología sociedad para elevar la satisfacción de los clientes del E-government

Los objetivos específicos son:

I. Sistematizar los fundamentos históricos - metodológicos para la generación de plataformas eficientes que permitan satisfacer las demandas de los clientes.

II. Diagnosticar el estado actual de las plataformas digitales gubernamentales de los GADs

III. Identificar las acciones para elevar la satisfacción de los clientes que utilizan las plataformas digitales gubernamentales de los GADs

\section{Metodología}

Para el desarrollo de la investigación bajo una concepción dialéctico - materialista como método general se combinaron métodos teóricos y empíricos; tales como:

Entre los métodos teóricos se encuentran el histórico-lógico, para el estudio y análisis que permitan la mejora continua de los procesos con énfasis en la satisfacción de los clientes, de las bases conceptuales y características de las mismas. Se utilizó el uso del análisis-síntesis, lo que permitió, de manera muy particular, después de un profundo análisis de valiosos referentes, resumir la información necesaria para la investigación en cuestión. En el caso del método inductivo-deductivo se logró profundizar en el estudio del tema de investigación desde lo general a lo particular. 
En relación a los métodos del nivel empírico se aplicó la revisión de documentos, para corroborar el nivel de cumplimiento y utilización de lo normado en los documentos básicos y otros productos elaborados en la propia dinámica de los procesos. La observación para la identificación del estado actual de la calidad de servicios que brinda las plataformas digitales de los GADs

Se utilizó la encuesta para conocer el grado de satisfacción de los clientes que utilizan la plataforma digital de los GADS en cuanto a la calidad y funcionalidad del servicio que se oferta.

\section{Resultados}

Para comprender como incluye la innovación tecno científica desde la perspectiva de los estudios de la ciencia, tecnología y sociedad (CTS), es necesario partir del entendimiento del termino desarrollo local en este ámbito Guzón, A (2005) al plantearse el "desarrollo local como trayectorias específicas de desarrollo que se configuran en elementos históricos, geográficos y en mentalidades, pero que no están totalmente predeterminadas sino que se transforman y evolucionan a partir de la prácticas de los propios actores, combinada con circunstancias y coyunturas que lo favorecen", unido a elementos que incorpora Núñez, J (2006) a la definición alrededor del tema al asumir el desarrollo local como "la realización del potencial de cambios cualitativos que se dan en estos escenarios y donde el factor conciencia, en el uso eficiente de los recursos endógenos, puede favorecer el mejoramiento de la calidad de vida"

"El desarrollo económico local constituye un proceso activador de la economía y dinamizador de la sociedad local, que mediante el aprovechamiento fundamentalmente de los recursos endógenos existentes en un determinado territorio, se pone en capacidad de estimular y fomentar su crecimiento económico, creando por tanto empleo y riqueza y poniendo éstas en función de mejorar la calidad de vida y la satisfacción de las necesidades siempre crecientes de las comunidades locales.

Es un proceso mediante el cual, los gobiernos locales establecen iniciativas y promueven actividades económicas eficientes y eficaces, de forma coordinada con todos los agentes políticos y sociales, en proyectos conjuntos que influyen decisivamente en el sector productivo, incentivándolos con el objeto principal de rediseñar la estructura socioeconómica del territorio, en función de pasar de los niveles primarios a niveles secundarios, terciarios o cuaternarios de desarrollo y así incrementar los valores productivos, la eficiencia en la gestión y la efectividad social" (Lazo Vento, Carlos, 2002).

En este mismo ámbito es fundamental identificar que los estudios de "ciencia, tecnología y sociedad" (CTS) constituyen un campo de trabajo académico y de investigación, con una creciente implantación a nivel internacional, que tiene por objeto el estudio de la naturaleza social del conocimiento científico-tecnológico y sus incidencias en los diferentes ámbitos económicos, sociales, ambientales, culturales, etc. 
Para comprender la importancia de este fenómeno científico-tecnológico en su contexto social, tanto en relación con sus condicionantes sociales como en lo que concierne a sus consecuencias sociales y ambientales, es importante tener una breve y puntual panorámica fidedigna referente a los estudios CTS, alrededor del mundo.

Los estudios CTS se origina hace tres décadas es decir a "Finales de los 60 y principios de los 70, son también los años del surgimiento de (...) iniciativas como los Estudios Sociales de la Ciencia y la Tecnología (...) Con un planteamiento contextualizado e interdisciplinario, los estudios CTS se centran en el análisis de los aspectos sociales de la ciencia y la tecnología, tanto en lo concerniente a sus antecedentes sociales como en lo que atañe a sus consecuencias sociales y ambientales" (López Cerezo, 2001: 15).

La trayectoria de los estudios de ciencia tecnología y sociedad es también la historia de una reacción critica, en los ámbitos académicos y educativo, contra la imagen clásica de la ciencia, una imagen esencialista y triunfalista, y de sus relaciones con la tecnología y la sociedad.

Los atributos listados pueden ser suficiente para saber con exactitud hacia donde se orientan los proyectos de innovación tecno científica al momento de generar plataformas digitales con el objetivo de fortalecer los gobiernos digitales, no obstante, definir algunos tipos no quedaría fuera de lugar. Se considera necesario porque según sean éstos, varían los grados de riesgo en su ejecución, así como los grados de libertad que se tienen para su implementación (Pmbok 1996, Heredia 1995)

La fase siguiente en el continuo se tiene en los proyectos correspondientes a la construcción de las plataformas digitales que sincronicen la funcionalidad administrativa y a la vez fomente fortaleza a los pilares del desarrollo sostenible. En este ámbito el grado de libertad de que se dispone en ese tipo de proyecto es todavía menos que otros vinculados a la generación de desarrollo local ya que este fundamenta su accionar en la funcionalidad eficiente de institucionalidad entorno al sector administrativo de recursos, puesto que normalmente, ya se conocerá tanto el coste de los recursos por cada unidad de los mismos que intervengan, la cantidad necesaria de cada recurso y también la naturaleza de los mismos.

Este tipo de actividades del continuo representan el límite superior de lo que entendemos por Proyecto, ya que cuando el sistema se ha construido, y entra en una fase normal de explotación, ya debe dirigirse mediante otra metodología, puesto que formará parte de una de las actividades normales de los GADs destinadas a hacerla cumplir su finalidad de sobrevivir a lo largo del tiempo.

A lo largo de esta investigación, se darán unas interfaces entre las distintas que hemos ido definiendo. Así, puede haber alguna interface entre investigación básica e investigación aplicada, lo mismo que entre ésta y los proyectos de investigación y desarrollo; también es frecuente que ésta se produzca entre la I+D vinculando al desarrollo local.

Se pueden considerar proyectos a los efectos de todo lo aquí tratado, los que corresponden 
a Investigación Aplicada los que corresponden a Investigación y Desarrollo (I+D), los de construcción física de los sistemas, no así, aquellas actividades que tengan relación directa con la investigación básica.

Con respecto a las actividades de automatización, debe señalarse que dentro de las mismas también existe el concepto de Proyecto. Por una parte, pueden considerarse Proyectos, por cumplir con su definición, la producción o generación de enlaces internos y externos que fomente institucionalidad. Igualmente, se aplica el concepto Proyecto en el diseño de prototipos enlazados con fases de producción; estas actividades que permiten el solape de distintas fases de la producción, se tratan actualmente con las metodologías de Dirección de Proyecto.

La gestión de la administración pública es determinante en la búsqueda del desarrollo local y los gobiernos deben jugar el rol de conector indispensable para la interacción proactiva entre los diferentes actores.

Facilitar las interrelaciones entre aspectos económicos, políticos, institucionales y culturales integrados en redes de actividades innovadoras, aún como condición de un desarrollo que incorpore fuertes componentes externos al propio territorio, pero con visión estratégica desde lo local Boffill Vega (2008). Los autores de la investigación consideran también el aspecto social entre las interrelaciones para promover el desarrollo local.

En esta misma línea, la calidad y su evaluación ha evolucionado por diferentes períodos hasta llegar a la gestión total de calidad, donde la responsabilidad es tanto de la dirección (en lo referente a procedimientos, métodos de trabajo, materiales, maquinarias, formas de organización) como del trabajador de línea que debe responder por su actuación y resolver las dificultades a su nivel, lo que implica a toda la organización en la satisfacción de oferentes y demandantes

Al decir de Gutiérrez Pulido (1996) la calidad total no es trabajar más, sino en forma más inteligente, armoniosa, viene de mejorar los procesos productivos y administrativos . El equipo ASH $(1999,2000)$ considera que el enfoque de la gestión total de la calidad implica su articulación con la gestión de recursos humanos como ejes vertebradores, sin los cuales sería imposible conseguir la excelencia empresarial.

En el estudio de la calidad del servicio constituyen referentes obligatorios los llamados "gurúes de la calidad" (Cantú, 1997), las escuelas europea y norteamericana que han marcado pautas de imprescindible análisis.

Para la mayoría de los autores la calidad es vista a través de la discrepancia percepciónexpectativas (Gronroos, 1988, 1994; Parasuraman, Zeithaml y Berry, 1988, 1991; Valls Figueroa, 2006; Parra Feríe, 2005), así como para los modelos conceptuales: Malcolm Baldrige (1987) , Servman (1987, 1988, 1990, 1994), EFQM (1991), Modelo Iberoamericano de Excelencia (1999). Sin embargo, otros autores y modelos analizan la calidad solo a través de la percepción (Jonson, Tiros, Lancioni, 1987; Cronin y Taylor, 
1992; Teas, 1993; Cuétara y Frías, 1996; Noda Hernández, 2004; Pérez Campdesuñer, 2006).

Por tanto los autores consideran que el estudio de la calidad a través del paradigma de la disconformidad (percepción-expectativas) es correcto, además de que permite la búsqueda de las necesidades explícitas e implícitas, sin embargo, la manera de recoger la información sobre las expectativas pueden viciar esta importante información, pues en la mayoría de los casos se realiza después que el CE ha comenzado a recibir el servicio; la propuesta de Valls Figueroa (2006) con el modelo Servqual modificado es la opción más aceptada consultada hasta el momento para suplir esta observación

La medición de las expectativas en la atención de los servicios de las plataformas digitales de los GADs, es una tarea compleja y difícil de realizar, aunque los autores comparten la importancia de su estudio en cualquier servicio que se brinde al cliente.

Esta situación se debe a: que estos servicios profesionales altamente complejos, al cumplir una función social y lucrativa, el nivel de exigencia del cliente es mucho más alto que en otros servicios no lucrativos, la tolerancia del cliente es menos amplia y los servicios adecuados (el aceptado) y esperados presentan un nivel más alto, unido a que la mayoría de los CE han tenido una experiencia anterior con este tipo de servicio, ya sea en el mismo centro o en otro, lo que mediatiza sus expectativas ; además el cliente no conoce las peculiaridades del servicio, por lo que sus expectativas se limitan a algunos aspectos tangibles, de empatía, de capacidad de respuesta y seguridad (confort, limpieza, organización del área, trato amable, respetuoso, rapidez en la atención, elementales que no deciden realmente la calidad de la atención al cliente.

Al mismo tiempo, el conocimiento de las expectativas del cliente, en las condiciones actuales no permite mejorar el servicio en función de ellas, no se gestiona según las expectativas. Lo más importante en este caso a criterio de los autores es conocer la calidad percibida de ambos clientes, su relación, así como los elementos científico-técnicos de la calidad de la atención, aunque se considera que, en investigaciones posteriores, donde el desarrollo del sector y de la actividad turística sea mayor puede tenerse en cuenta el análisis de las expectativas para poder accionar la organización en función de ellas.

El autor del presente trabajo conceptualiza la calidad percibida como un proceso psicológico mediante el cual se produce un reflejo integral de varios elementos asociados al servicio en forma de imágenes concretas e inmediatas, se manifiesta mediante juicios del cliente sobre la excelencia de una entidad en la que se satisfagan o no las necesidades del cliente.

En la vida laboral y social del hombre está presente la necesidad de la adquisición del conocimiento, el deseo de saber algo, de conocer la forma de solucionar un problema determinado, por lo que para alcanzar el resultado deseado o su solución se requieren esfuerzos y dedicación.

La mayor parte de la investigación referido al término de conducta es planteada 
certeramente por Robbins S.(1998) en su Obra” Comportamiento Organizacional "y se ha interesado en tres actitudes:" Compromiso con el trabajo, satisfacción laboral y compromiso organizacional.

En este ámbito es fundamental determinar el Compromiso con el Trabajo, lo define como el grado en el cual una persona se identifica con su trabajo, participa activamente en él y considera su desempeño importante para la valoración propia.

Igualmente, las satisfacciones laborales, es la actitud del trabajador frente a su propio trabajo, dicha actitud está basada en las creencias y valores que el trabajador desarrolla de su propio trabajo.

Las actitudes son determinadas conjuntamente por las características actuales del puesto como por las percepciones que tiene el trabajador de lo que "deberían ser".

Herramienta estratégica indispensable para enfrentar los nuevos desafíos que impone el medio. Es impulsar a nivel de excelencia las competencias individuales, de acuerdo a las necesidades operativas. Garantiza el desarrollo y administración del potencial de las personas, "de lo que saben hacer" o podrían hacer. [Cecilia Parra]

En este concepto se reúnen todos los aspectos a nuestro juicio son esenciales en el mismo porque incluye como el trabajador puede adquirir sus conocimientos para realizar su labor de forma competente y además como esta preparación puede contribuir al fortalecimiento de sus valores y su modo de actuación.

Caracterización del estado actual del proyecto de plataformas eficientes que permitan satisfacer las demandas de los clientes.

El proyecto de plataformas eficientes que permitan satisfacer las demandas de los clientes de los GADs de la provincia de Chimborazo toma mayor impulso raíz de la pandemia

Su objetivo se basa en el incremento de servicios digitales a través del internet con la finalidad de evitar las aglomeraciones

Su misión se centra en generar eficiencia y eficacia en los procesos administrativos los cual se refleja en bajar costos y tiempos dándole mayor fortaleza a la institucionalidad y dinámica administrativa generando en los clientes cumplimiento de responsabilidades y rapidez en los procesos.

En la mayoría de los GADs la automatización informática de los servicios institucionales ha obtenido resultados satisfactorios en cuanto a los resultados económicos, presenta rentabilidad en cuanto a los demás indicadores de eficiencia.

En este sentido se plantea acciones para elevar la satisfacción de los clientes en el uso de las plataformas digitales para el desarrollo, para lo cual se presenta el diagnóstico que reveló el estado actual del sistema informático institucional acorde a su capacidad tecnológica donde se utilizaron como métodos la revisión de documentos, la observación y la encuesta. Luego de este estudio diagnóstico, se presenta el plan de acciones para la 
elevación de la satisfacción de los clientes del E-government

\section{Resultados de la aplicación de la revisión de documentos y software}

Se revisaron documentos sobre la sistematización, la estructura de la producción, el plan técnico económico, el plan de prevención de riesgo y los controles de calidad de las producciones, tal como se muestra a continuación:

Objetivo: Revisar el estado actual de indicadores que miden la efectividad del proyecto y que inciden en la satisfacción de los clientes

Guía de revisión de documentos

- $\quad$ Plan de producción de la fábrica.

- La estructura de la producción.

- El plan técnico económico.

- El plan de prevención de riesgo

- Los controles de calidad de las producciones.

Se pudo determinar la mayoría de los GADs presenta una buena planificación en cuanto a la búsqueda de la automatización, no siendo así en la práctica donde todavía se encuentran algunas alteraciones ya que se le está ya que no se hizo un estudio de los clientes cuentan con las TICs, factor este determinante en la calidad de sus productos a partir de que se afectan las características organolépticas de la producción terminada.

Se cuenta con un plan técnico-económico actualizado, con una desagregación ajustado a los niveles de actividad de los GADs y a las necesidades de desarrollo de la misma para el presente año y el futuro de la misma. Además, se pudo comprobar que la unidad no cuenta con un programa para la implementación de las acciones encaminadas a la protección del consumidor, así como no evidencia la efectividad de las acciones plasmadas en el plan de prevención de riesgos.

En relación a los controles de la calidad de las producciones se pudo constatar que estos se realizan, según lo planificado. En los documentos revisados aparecen entre las principales dificultades que se han detectado violaciones en el cumplimiento de los procedimientos para los procesos tecnológicos entre las que se destacan que violaciones del proceso productivo a la hora de la elaboración del pan y el coscorrón ya que no se realiza correctamente el proceso de mezclado de las materias primas así como existen alteraciones con la temperatura adecuada para el tiempo de la fermentación y que no se pesa el producto agua, materia prima esta de suma importancia en la elaboración del producto terminado.

En el ámbito de los resultados del método de observación para la aplicación de la guía de observación tal como se observa a continuación:

Objetivo: Identificar el estado actual del cumplimiento de los procedimientos que se aplican durante el proceso de producción de las plataformas digitales gubernamentales de 
los GADs

- Quién emite la orden de registro

- Cómo se realiza el pre-despacho.

- Cómo se sistematiza el proceso de interacción de la digitalización de servicios.

- Qué tiempo y que procesos se automatizo

- A qué sistemas prefiere la población se automatice

- Qué tiempo permanecen o se demoran las personas usando las plataformas interactivas de los GADs

- $\quad$ Con que frecuencia se satura el servicio por falencias en el sistema informático.

- Cuánto tiempo demora el cliente por proceso automatizado

- Con qué frecuencia el administrador y el técnico de calidad realizan un muestreo de fiscalización y evaluación.

A partir de estos análisis se pudo corroborar que existen violaciones que afectan considerablemente la calidad del sistema digital o en línea interactivo planteado y estas a la vez trae consigo insatisfacciones de los clientes del proyecto que consumen las mismas.

En este ámbito, la valoración sintetizada de las principales deficiencias detectadas nos permite determinar que:

No todos los instrumentos y mecanismo tecnológicos están al alcance de los usuarios para darle dinamismo a los procesos tecnológicos implementaos

No se ha realizado estudios socioeconómicos por sectores para determinar la capacidad de la población y de los GADs por territorio para implementar y adquirir dispositivos y software electrónicos o sistemas computarizados

Existen parámetros poco prácticos implementados en las plataformas como producto del poco análisis de validación en las mallas de sustentación teórica que son base de los procesos digitales

No se consideró la capacidad de carga que recibirá el sistema por lo cual tiende a saturarse y bloquea los procesos productivos

Como resultados de la aplicación de la encuesta para la medición de la satisfacción de los clientes ante esta iniciativa tecno científica de modernizar la administración utilizando sistemas informáticos que permitan interactuar y generar servicios en línea entre los clientes y la institucionalidad de los GADs para Desarrollo Local, sobre la muestra poblacional por territorio se determinó que:

Con relación a la calidad de los servicios que brinda la fábrica de la muestra que el $65 \%$ de los encuestados manifestaron que la calidad es regular; el $25 \%$ manifestaron que es buena y solamente el $10 \%$ manifiestan que la calidad es muy buena.

A partir de la tabulación de las encuestas aplicadas para conocer el criterio de los consumidores sobre la calidad del servicio en línea se pudo identificar que el $76 \%$ de los 
encuestados manifiestan que la calidad es regular y 12 encuestados, el $24 \%$, refleja que la calidad es buena lo que evidencia que existen dificultades con la calidad del servicio en línea que se oferta que se ofertan.

En relación a la sistematicidad con la que se oferta el producto, como resultado de la encuesta se obtuvo que el $80 \%$ de los encuestados tiene problema con la saturación de la pág. web y el $20 \%$ expresa que se da rara vez la saturación el $100 \%$ manifiesta que casi nunca a excepción de lo días pico de cumplimiento de obligaciones

Para enfrentar las dificultades antes encontradas a partir de la aplicación de los diferentes métodos empíricos aplicados, se elaboró un plan de acción para la elevación de la satisfacción de los clientes del proyecto de iniciativa tecno científica aplicada a los GADs para el Desarrollo Local, se plantean acciones para la elevación del nivel de satisfacción de los clientes como:

- Desarrollar seminarios sobre las buenas prácticas en el uso y generación de plataformas digitales.

- Realizar actividad práctica temporales sobre la base del uso y aplicación de sistemas

- Verificar el plan de temas y las sistematicidades de los procesos.

- Controlar la efectividad del especialista de calidad en el diseño del software.

- Comprobar de forma sorpresiva el peso y la calidad del servicio para evitar saturaciones

- Verificar las acciones del administrador del software para mejorar la calidad de las producciones.

- Constatar en la viabilidad de los procesos de fiscalización y de evaluación técnica y social sobre el servicio

\section{Conclusiones}

- La sistematización de los referentes teóricos sobre los proyectos de desarrollo local y la satisfacción de los clientes permitieron a los autores y críticos de procesos una mejor comprensión alrededor del tema.

- La encuesta de satisfacción de los clientes, la observación y la revisión de documentos del proyecto aplicados arrojaron información valiosa sobre los resultados alcanzados hasta la fecha y el estado actual de satisfacción de los clientes del proyecto de innovación tecno científica aplicada a nivel de GADs; en particular que existe una fuerte relación entre la calidad de los productos que se ofertan y la satisfacción de los clientes.

- Se pudo identificar las acciones necesarias para satisfacer a los clientes a partir de sus insatisfacciones declaradas durante la investigación. El éxito radicará en la responsabilidad con la que se asuma su implementación. 


\section{Bibliografía}

Alburquerque LLorents, F. (1994). "Reflexiones sobre el Futuro del Mundo Rural en Andalucía". Boletín de Información Económica N 12. España.

Alburquerque LLorents, F. (1995). "Espacio, Territorio y Desarrollo Económico Local". Publicaciones ILPES. Chile.

Beaudoux, E. y Colectivo. (1992). "Guía Metodológica de Apoyo a Proyectos y Acciones para el Desarrollo". "De la Identificación a la Evaluación”. IEPALA. España.

Barredo Medina, L. 1996. "Democracia sociedad civil y gobernabilidad en Cuba". ANPP. Cuba.

Blanco-Díaz, Elena (2014): Obstáculos a vencer en el desarrollo local. Estudio de caso: municipio Guanabacoa; en: Econ. y Desarrollo vol.151 no.1 La Habana ene.jun. 2014; disponible

en: http://scielo.sld.cu/scielo.php?script=sci_arttext\&pid=S025285842014000100015 [Consultado: 22.05.2017].

Boffill Vega, C. Sinaí; Reyes Fernández; C. Rafael; Calcines Díaz, Carlos; Luna Piedad, Carlos (2015): Gestión del Gobierno local con apoyo en el conocimiento y la innovación. Lecciones aprendidas en Yaguajay, Cuba; en: Retos de la Dirección;

$(1) / 2015$;

p.201-217;

disponible

en: http://scielo.sld.cu/scielo.php?script=sci_arttext\&pid=S230691552015000100010 [Consultado: 22.05.2017].

Del Cano, Alfredo y de la Cruz, Pilar (1995). Conceptos Básicos de la Dirección de Proyectos. UNED, Madrid, España.

Hernández Junco, Varna (2009). Evaluación y mejora de la actuación del personal y su incidencia en la calidad del servicio asistencial hospitalario. Tesis presentada en opción al grado científico de Doctor en Ciencias Técnicas. Universidad de Matanzas. Cuba

Gómez Prieto, Jorge Luis (2014): Desarrollo Local. Alternativa viable para la sostenibilidad del desarrollo socioeconómico en Cuba; en: Observatorio iberoamericano del desarrollo local y la economía social; Revista académica, editada y mantenida por el Grupo EUMED.NET de la Universidad de Málaga, Nro.16; Junio de 2014; disponible en: www.eumed.net/rev/oidles/16/desarrollocuba.pdf [Consultado: 24.11.2015].

Guzón Camporredondo, Ada; Hernández Márquez (2015): A propósito del desarrollo local en Cuba; en: Pérez Hernández, Lissette; Díaz Legón, Orestes J. (Coordinadores, 2015): ¿Qué municipio queremos? Respuestas para Cuba en clave de descentralización y desarrollo local; La Habana: Editorial UH, 2015, p.105-116. 

septiembre, 2021

Núñez Jover, J. (2014). Universidad, conocimiento, innovación y desarrollo local. Editorial Universitaria Felix Varela. La Habana. 


\section{PARA CITAR EL ARTÍCULO INDEXADO.}

Fonseca Vasconez, J. F., Andrade Álvarez, C. E., \& Viteri Ojeda, J. C. (2021). Innovación tecnocientífica para el fortalecimiento del E-government una apreciación desde el enfoque de los estudios de la ciencia - tecnología - sociedad . AlfaPublicaciones, 3(3.2), 81-94. https://doi.org/10.33262/ap.v3i3.2.105

\section{\Ciencia}

El artículo que se publica es de exclusiva responsabilidad de los autores y no necesariamente reflejan el pensamiento de la Revista Alfa Publicaciones.

El artículo queda en propiedad de la revista y, por tanto, su publicación parcial y/o total en otro medio tiene que ser autorizado por el director de la Revista Alfa Publicaciones.
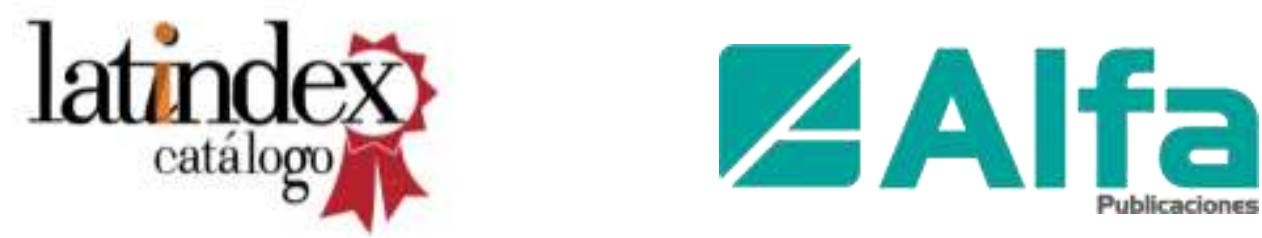

1 